\title{
Rational Cubic Spline Interpolation for Monotonic Interpolating Curve with $C^{2}$ Continuity
}

\author{
Samsul Ariffin Bin Abdul Karim ${ }^{1, *}$ \\ ${ }^{1}$ Fundamental and Applied Sciences Department, Universiti Teknologi PETRONAS, Bandar Seri \\ Iskandar, 32610 Seri Iskandar, Perak DR, Malaysia
}

\begin{abstract}
Monotonicity preserving interpolation is very important in many sciences and engineering based problems. This paper discuss the monotonicity preserving interpolation for monotone data set by using $C^{2}$ rational cubic spline interpolant (cubic/quadratic) with three parameters. The data dependent sufficient conditions for the monotonicity are derived with two degree freedom. Numerical results suggests that the proposed $C^{2}$ rational cubic spline preserves the monotonicity of the data and outperform the performance of the other rational cubic spline schemes in term of visually pleasing.
\end{abstract}

\section{Introduction}

Visualizing the scientific data is important in computer graphics and geometric modeling areas. One of the requirements for scientific visualization is that the ability of the interpolating scheme to preserves the geometric properties of the data sets. For instance if the given data sets are monotone, then the resulting interpolating curves also must be monotonic on the entire given interval. Some examples of monotonic data includes the population at one country as well as the approximation of couples and quasi couples in statistics (Karim and Kong [13]). The usual $C^{2}$ cubic spline interpolation is very smooth but for shape preserving interpolation purpose, the cubic spline are unable to preserves the shape of the data on entire given interval resulting the interpolating curve may be oscillates on some interval. Fritsch and Carlson [8] constructed the monotonic cubic Hermite spline polynomial to preserve monotone data set. Their scheme have some drawback such as it require the modification of the first derivatives if shape violation is found. Fritsch and Carlson [8] scheme is not recommended for monotonicity preserving interpolation. Thus one of the alternative strategy was the used of rational cubic spline for monotonicity preservation.

There exist many research papers on the construction of rational cubic spline for monotonicity preserving interpolation. Some literature reviews are as follows: Delbourgo and Gregory [6,7] have discussed the monotonicity and convexity preservation by using rational cubic spline (quadratic denominator) with one parameter. Sarfraz [18], Sarfraz et al. [19] and Abbas et al. [1] also studied the use of rational cubic interpolant for preserving the monotone data. Meanwhile Hussain and Hussain [11] and Hussain et al. [12] studied

* Corresponding author: samsul_ariffin@utp.edu.my 
monotonicity preserving for curves and surfaces by utilizing rational cubic spline with quadratic denominator- without any degree freedom i.e. no free parameters for shape modification. In Sarfraz et al. [19] the rational cubic spline with quadratic denominator has been used for monotonicity with $C^{2}$ continuity - without any degree freedom. Sarfraz [18] also discussed the used of rational cubic spline (cubic/cubic) for monotonicity preserving with $C^{2}$ continuity but his schemes also not received much attention since there are no degree freedom. Similarly the rational cubic spline of Gregory [9] also did not have any degree freedom i.e. free parameters for shape modification. Abbas et al. [1] discussed the monotonicity by using $C^{2}$ rational cubic spline with two free parameters. This paper is a continuation from our previous works (Karim and Kong [14]). The rational cubic spline with $C^{2}$ continuity will used for monotonicity-preserving interpolation for functional data. The main contribution this paper is summarized as follows:

(i) Our scheme give comparable results compare with existing schemes. Indeed for some data set, our scheme is better.

(ii) Our scheme not involving any knots insertion as appear in the works of Lahtinen [15], Lam [16], Schumaker [21], Fristch and Carlson [8], Schmidt and Hess [20] and Butt and Brodlie [5].

(iii) Our scheme has two degree freedom meanwhile there are no degree freedom in the works of Sarfraz [18], Delbourgo and Gregory [6,7] and Gregory [9].

\section{$2 C^{2}$ rational cubic spline interpolant}

This section gives the definition for $C^{2}$ rational cubic spline interpolant with three parameters. The mathematical derivations are taken from Karim and Kong [14]. For simplicity, we assume that the data points $\left\{\left(x_{i}, f_{i}\right), i=0,1, \ldots, n\right\}$ are given such that $x_{0}<x_{1}<\ldots<x_{n}$. Let $h_{i}=x_{i+1}-x_{i}, \Delta_{i}=\left(f_{i+1}-f_{i}\right) / h_{i}$ and $\theta=\left(x-x_{i}\right) / h_{i}$ such that $0 \leq \theta \leq 1$. For $x \in\left[x_{i}, x_{i+1}\right], i=0,1,2, \ldots, n-1$ the rational cubic spline interpolant with three parameters is defined as follows:

$$
s(x) \equiv s_{i}(x)=\frac{A_{i 0}(1-\theta)^{3}+A_{i 1} \theta(1-\theta)^{2}+A_{i 2} \theta^{2}(1-\theta)+A_{i 3} \theta^{3}}{(1-\theta)^{2} \alpha_{i}+\theta(1-\theta)\left(2 \alpha_{i} \beta_{i}+\gamma_{i}\right)+\theta^{2} \beta_{i}}
$$

with $A_{i j}, j=0,1,2,3$ as:

$$
\left\{\begin{array}{l}
A_{i 0}=\alpha_{i} f_{i}, \\
A_{i 1}=\left(2 \alpha_{i} \beta_{i}+\alpha_{i}+\gamma_{i}\right) f_{i}+\alpha_{i} h_{i} d_{i}, \\
A_{i 2}=\left(2 \alpha_{i} \beta_{i}+\beta_{i}+\gamma_{i}\right) f_{i+1}-\beta_{i} h_{i} d_{i+1}, \\
A_{i 3}=\beta_{i} f_{i+1} .
\end{array}\right.
$$

$C^{2}$ continuity i.e. $s^{(2)}\left(x_{i+}\right)=s^{(2)}\left(x_{i-}\right), \quad i=1,2, \ldots, n-1$, lead us to the following system of linear equations:

with

$$
a_{i} d_{i-1}+b_{i} d_{i}+c_{i} d_{i+1}=e_{i}, \quad i=1,2, \ldots, n-1
$$




$$
\begin{aligned}
& a_{i}=h_{i} \alpha_{i-1} \alpha_{i} \\
& b_{i}=h_{i} \alpha_{i}\left(\gamma_{i-1}+2 \alpha_{i-1} \beta_{i-1}\right)+h_{i-1} \beta_{i-1}\left(\gamma_{i}+2 \alpha_{i} \beta_{i}\right) \\
& c_{i}=h_{i-1} \beta_{i-1} \beta_{i} \\
& e_{i}=h_{i} \alpha_{i}\left(\gamma_{i-1}+\alpha_{i-1}+2 \alpha_{i-1} \beta_{i-1}\right) \Delta_{i-1}+h_{i-1} \beta_{i-1}\left(\gamma_{i}+\beta_{i}+2 \alpha_{i} \beta_{i}\right) \Delta_{i}
\end{aligned}
$$

The system of equation in (3) gives $n-1$ linear equations for $n+1$ unknown derivative values. By imposing (4) and (5), then the system of linear equations in (4) is strictly tridiagonal and has a unique solution for the unknown derivative parameters $d_{i}, i=1,2, \ldots, n-1$ for all $\alpha_{i}, \beta_{i}>0, \gamma_{i} \geq 0$. This solution can be found by using Thomas algorithm.

$$
\begin{aligned}
& s^{(1)}\left(x_{0}\right)=d_{0} \\
& s^{(1)}\left(x_{n}\right)=d_{n}
\end{aligned}
$$

\section{Sufficient condition for monotonicity}

The monotonicity preserving by using $C^{2}$ rational cubic spline will be further elaborate in this section. Given that the monotone data sets $\left(x_{i}, f_{i}\right), i=0,1, \ldots, n$ such that $x_{0}<x_{1}<\ldots<x_{n}$. For a monotonic increasing (decreasing), the necessary condition for monotonicity is:

$$
f_{0} \leq f_{1} \leq \ldots \leq f_{n}\left(\text { or } f_{0} \geq f_{1} \geq \ldots \geq f_{n} \text { for monotonic decreasing }\right)
$$

Condition (6) is equivalent to

$$
\Delta_{i} \geq 0 \quad \text { (or } \Delta_{i} \leq 0 \text { for monotonic decreasing data) }
$$

The necessary and sufficient condition for the $C^{2}$ monotonicity of rational cubic spline cubic interpolant will be derived by finding the conditions on parameter $\gamma_{i}$ and it is data dependent. For monotonic increasing rational cubic interpolant $s(x)$ in (1), the first derivative must satisfy the following inequalities:

$$
d_{i} \geq 0, i=0,1,2, \ldots, n
$$

The rational cubic spline, $s(x)$ is monotonic increasing if and only if

$$
s^{(1)}(x) \geq 0, x_{0} \leq x \leq x_{n}
$$

The first derivative of the rational cubic spline interpolant $s(x)$ is given in Karim and Kong [13].

Theorem 1. Given a strictly monotonic increasing set of data satisfying (7), there exists a class of monotonic rational (of the form cubic/quadratic) interpolating spline $s(x) \in C^{2}\left[x_{0}, x_{n}\right]$ involving free parameters $\alpha_{i}$ and $\beta_{i}$ provided that if it satisfy the following sufficient conditions:

$$
\begin{gathered}
\alpha_{i}>0, \beta_{i}>0, \\
\gamma_{i}=\delta_{i}+\operatorname{Max}\left\{0, \beta_{i}\left(\frac{d_{i+1}}{\Delta_{i}}-2 \alpha_{i}\right), \alpha_{i}\left(\frac{d_{i}}{\Delta_{i}}-2 \beta_{i}\right)\right\}, \delta_{i}>0, \Delta_{i}>0 .
\end{gathered}
$$


Remark 1: If the data are constant on certain interval $\Delta_{i}=0$, then setting $d_{i}=d_{i+1}=0$, will produce $s(x)=f_{i}=f_{i+1}$ is a constant on the interval $\left[x_{i}, x_{i+1}\right], i=0,1,2, \ldots, n-1$.

\section{Numerical demonstrations}

In order to illustrate the monotonicity preserving interpolation by using the proposed $C^{2}$ rational cubic spline interpolation (cubic/quadratic), choose Akima [4] data set i.e. wave distortion data in experiment conducted in laboratory.

Table 1. A monotone data from Akima [4]

\begin{tabular}{|c|c|c|c|c|c|c|c|c|c|c|c|}
\hline$x_{i}$ & 0 & 2 & 3 & 5 & 6 & 8 & 9 & 11 & 12 & 14 & 15 \\
\hline$f_{i}$ & 10 & 10 & 10 & 10 & 10 & 10 & 10.5 & 15 & 50 & 60 & 85 \\
\hline$d_{i}$ & 0 & 0 & 0 & 0 & 0 & 0 & 1.083 & 24.083 & 25 & 18.333 & 31.667 \\
\hline
\end{tabular}

Table 2. Numerical results

\begin{tabular}{|c|c|c|c|c|c|c|c|c|c|c|c|}
\hline$i$ & 0 & 1 & 2 & 3 & 4 & 5 & 6 & 7 & 8 & 9 & 10 \\
\hline$d_{i}\left(C^{1}\right)$ & 0 & 0 & 0 & 0 & 0 & 0 & 1.083 & 24.083 & 25 & 18.333 & 31.667 \\
\hline$\Delta_{i}$ & 0 & 0 & 0 & 0 & 0 & 0.5 & 2.25 & 35 & 5 & 25 & \\
\hline$\alpha_{i}$ & - & - & - & - & - & 2 & 2 & 2 & 2 & 2 & \\
\hline$\beta_{i}$ & - & - & - & - & - & 2 & 2 & 2 & 2 & 2 & \\
\hline$\gamma_{i}$ & - & - & - & - & - & 0.25 & 13.66 & 0.25 & 0.25 & 0.25 & \\
\hline$d_{i}\left(C^{2}\right)$ & 0 & 0 & 0 & 0 & 0 & 0 & 0.747 & 17.498 & 25.388 & 14.815 & 31.667 \\
\hline
\end{tabular}

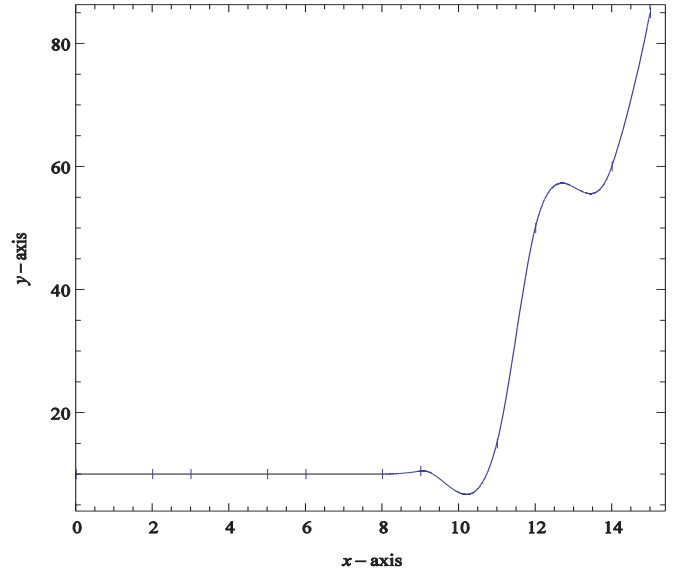

(a) Cubic Hermite spline

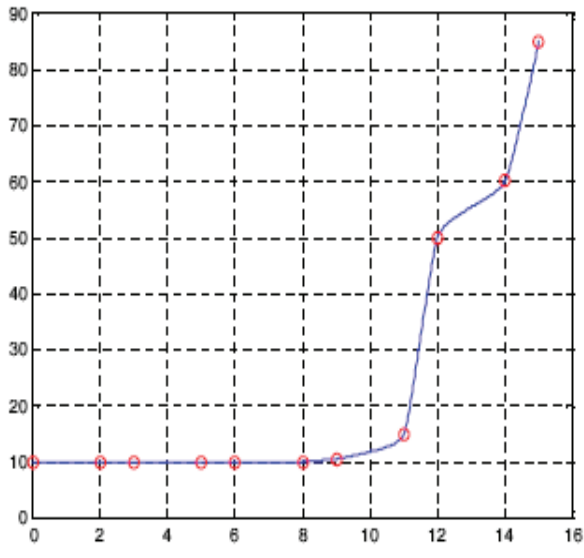

(b) From Sarfraz et al. [19] 


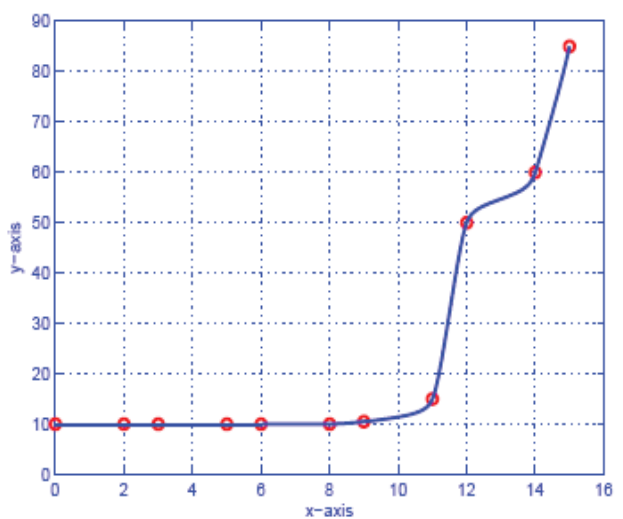

(c) From Abbas et al. [3]

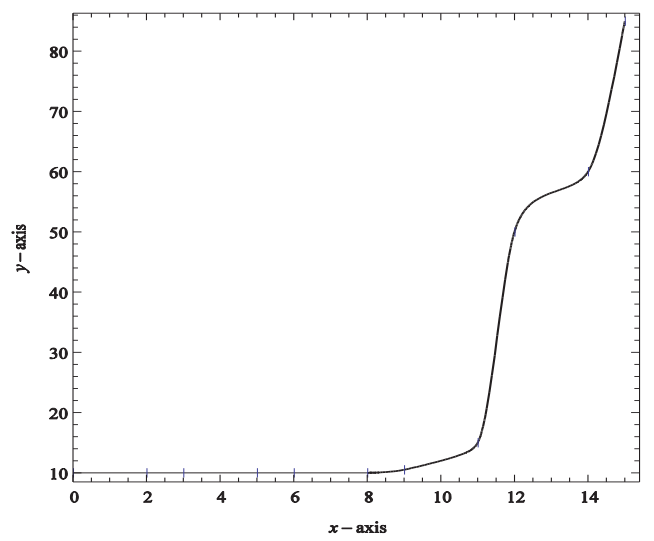

(e) The proposed scheme with $\alpha_{i}=\beta_{i}=0.5$

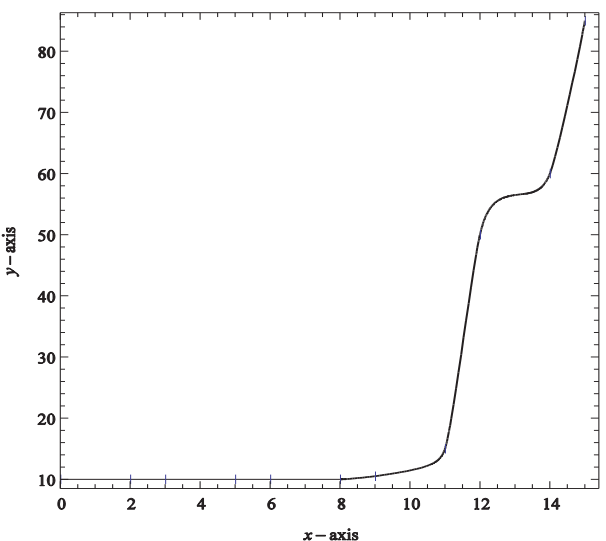

(d) The proposed scheme with $\alpha_{i}=\beta_{i}=2$

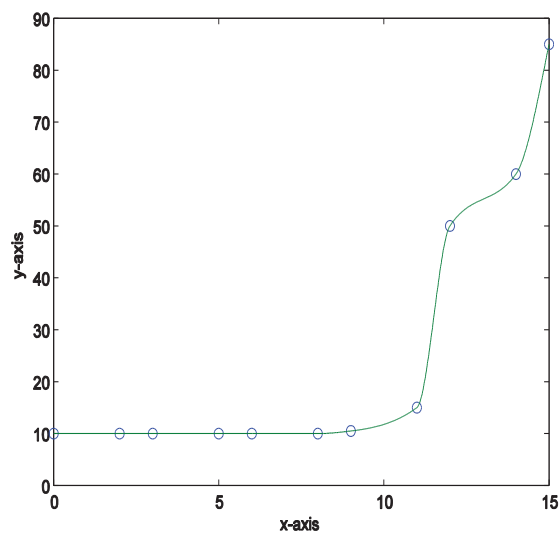

(f) PCHIP

Fig. 1. Comparison of interpolating curves

Fig. 1 shows the monotonic interpolating curves for monotone data sets given in Table 1 . Figs. 1(a)) shows the default cubic Hermite spline polynomial. Figs. 1(b) and 1(c) show the monotonic interpolating by using the rational spline schemes by Sarfraz et al. [19] and Abbas et al. [1] respectively. Fig. 1(d) is obtained by using the data in Table 2. From Figs. 1(d) and (e) the proposed $C^{2}$ rational cubic spline give smooth results compare to the works of Sarfraz et al. [19] and Abbas [3]. Meanwhile from Fig. 1(f), shows that PCHIP also can produce the monotonic interpolating curves but not visually pleasing and some part of interpolating curves very tight and the smoothness is only $C^{1}$.

\section{Discussions and Conclusion}

From all numerical results, it can be seen clearly that the proposed $C^{2}$ rational cubic spline works very well and it is comparable with existing schemes such as Abbas [4], Abbas et al. [1] and Sarfraz et al. [19]. Future work will be focusing on other shape preserving criteria.

Acknowledgement to Universiti Teknologi PETRONAS (UTP) for the financial support received in the form of research grants: Short Term Internal Research Funding (STIRF) No. 0153AA-D91 and YUTP: 0153AA-H24 including the Mathematica and MATLAB Softwares. 


\section{References}

1. M., Abbas, A.A. Majid and J.M. Ali. Applied Mathematics and Computation, 219: pp. 2885-2895 (2012).

2. U., Bashir and J.M. Journal of Applied Mathematics, Vol. 2013, Article ID 531497, 10 pages, (2013).

3. M., Abbas. Shape preserving data visualization for curves and surfaces using rational cubic functions [Ph.D. thesis], (School of Mathematical Sciences, Universiti Sains Malaysia, Penang,Malaysia, 2012).

4. H., Akima. J. Assoc. Comput. Mech. 17:589-602 (1970).

5. S., Butt and K.W. Brodlie. Computer and Graphics 17 (1):55-64 (1993).

6. R., Delbourgo and J.A. Gregory. SIAM J. Sci. and Statist. Comput. 6:967-976 (1985).

7. R., Delbourgo and J.A. Gregory. IMA Journal of Numerical Analysis, 5:397-406 (1985).

8. F.N., Fritsch and R.E. Carlson. SIAM J. Numer. Anal. 17: 238-246 (1980).

9. J.A., Gregory. Computer Aided Design 18(1):53-57 (1986).

10. M.Z., Hussain and M. Sarfraz. International Journal of Computer Mathematics, Vol. 86, No. 3, March 2009, pp. 423-430 (2009).

11. M.Z., Hussain and M. Hussain. Applied Mathematics and Computation 190, 1353-1364 (2007).

12. M.Z., Hussain, M. Sarfraz and T.S. Shaikh. World Applied Sciences Journal 16(11): 1496-1508 (2012).

13. F.M., Ibraheem, Hussain and M.Z. Hussain, The Scientific World Journal. Volume 2014, Article ID 602453, 14 pages, (2014).

13. S.A.A., Karim and V.P. Kong. Research Journal of Applied Sciences (RJAS), Volume: 9 , Issue: 4, Page No.: 214-223 (2014).

14. S.A.A., Karim and V.P. Kong. Journal of Applied Mathematics, Volume 2016 (2016), Article ID 4875358, 14 pages, (2016).

15. A., Lahtinen. Annals of Numerical Mathematics 3:151-161 (1996).

16. M.H., Lam. Virginia Journal of Science. Volume 41(1): 1-13 (1990).

17. S., Liu, Che, Z. and Zhu, Y. Mathematical Problems in Engineering, Volume 2015, Article ID 983120, 20 pages (2015).

18. M., Sarfraz. Computers \& Graphics 24(4):509-516 (2000).

19. M., Sarfraz, M.Z., Hussain, T.S., Shaikh and R., Iqbal. Data visualization using shape preserving $C^{2}$ rational spline. In Proceeding of $5^{\text {th }}$ International Conference on Information Visualisation, 2011, pp. 528-533 (2011).

20. J.W., Schmidt and W., Hess. BIT 28, 340-352 (1988).

21. L.L., Schumaker. SIAM J. Numerical Analysis 20:854-864 (1983). 